\title{
Detecting nonclassical primordial gravitational waves with Hanbury-Brown-Twiss interferometry
}

\author{
Sugumi Kanno ${ }^{1}$ and Jiro Soda ${ }^{2}$ \\ ${ }^{1}$ Department of Physics, Osaka University, Toyonaka 560-0043, Japan \\ ${ }^{2}$ Department of Physics, Kobe University, Kobe 657-8501, Japan
}

(Received 11 November 2018; published 9 April 2019)

\begin{abstract}
We consider the possible detection of nonclassicality of primordial gravitational waves (PGWs) by applying Hanbury-Brown-Twiss (HBT) interferometry to cosmology. We characterize the nonclassicality of PGWs in terms of sub-Poissonian statistics that can be measured by the HBT interferometry. We show that the presence of classical sources during inflation makes it possible to detect nonclassical PGWs with the HBT interferometry. We present two examples that realize the classical sources during inflation. It turns out that PGWs with frequencies higher than $10 \mathrm{kHz}$ enable us to detect their nonclassicality.
\end{abstract}

DOI: 10.1103/PhysRevD.99.084010

\section{INTRODUCTION}

One of the cornerstones of inflationary cosmology is that the large scale structure of the Universe has a quantum mechanical origin. Primordial gravitational waves (PGWs) from inflation could also arise out of quantum fluctuations. These invite the question of whether compelling observational evidence for the nonclassical nature of the initial fluctuations can be found.

The direct detection of gravitational waves in 2015 [1] encouraged us to find a way to address this question. Indeed, detecting PGWs is now an important target for gravitational wave physics $[2,3]$. They interact very weakly with matter, travel through the Universe virtually unimpeded, and hence give us information about the origin of the Universe. To detect them at present, the energy scale that generates them has to be around the grand unified theory scale in the conventional inflationary scenario. It is difficult to find a possible scenario other than the inflationary scenario to realize such a high energy scale. In this sense, if PGWs were detected, the detection could be regarded as a proof of inflationary cosmology. Recently, it was shown that the necessary energy scale for generating them can be reduced in the presence of gauge fields during inflation [4]. In this case, the PGWs have circular or linear polarization and then carry information about the model of inflation as well. Hence, besides the quantum mechanical origin of the Universe, they would tell us the inflationary scenario that the Universe experienced. On top of that, if we succeeded in detecting the

Published by the American Physical Society under the terms of the Creative Commons Attribution 4.0 International license. Further distribution of this work must maintain attribution to the author(s) and the published article's title, journal citation, and DOI. Funded by SCOAP . nonclassicality of PGWs, it would imply the discovery of gravitons.

In this work, we characterize nonclassicality by counting graviton numbers in a given state as is often done in quantum optics [5]. It is known that the particle number distribution for coherent fields is Poissonian. Namely, the mean number of gravitons is identical to its variance. In other words, the Fano factor (the ratio of variance to mean) equals 1 . Note that the distribution of gravitons in classical theory is always super-Poissonian and the Fano factor is above 1. Hence, subPoissonian distribution of graviton numbers or the Fano factor below 1 must be a signature of nonclassicality. The point is that the sub-Poissonian statistics can be detected with Hanbury-Brown-Twiss (HBT) interferometry [6,7].

In inflationary cosmology, the Bunch-Davies (BD) vacuum is usually assumed as the simplest initial state of quantum fluctuations of the Universe. The BD vacuum looks like a squeezed state [8] from the point of view of the radiation-dominated era of the Universe, and the graviton distribution of the squeezed state shows super-Poissonian. However, the latest Planck data show the possibility of deviation from the BD vacuum [9]. Thus, we consider a non-BD vacuum due to the presence of gauge fields as the initial state. Remarkably, this initial state becomes a squeezed coherent state and the graviton distribution of the squeezed coherent state shows sub-Poissonian. To predict the frequency range of nonclassical PGWs, we present two examples that realize the presence of gauge fields during inflation. We show that PGWs with frequency higher than $10 \mathrm{kHz}$ enable us to detect their nonclassicality with the HBT interferometry.

\section{QUANTUM INITIAL STATES OF PGWS}

We start by reviewing possible initial quantum states in the Universe [10]. 
The metric of tensor perturbations is expressed as

$$
d s^{2}=a^{2}(\eta)\left[-d \eta^{2}+\left(\delta_{i j}+h_{i j}\right) d x^{i} d x^{j}\right],
$$

where $\eta$ is the conformal time, $x^{i}$ are spatial coordinates, and $\delta_{i j}$ and $h_{i j}$ are the Kronecker delta and the tensor perturbations which satisfy $h_{i j}{ }^{j}=h^{i}{ }_{i}=0$. The indices $(i, j)$ run from 1 to 3 . To determine the scale factor $a(\eta)$, we assume the Universe goes through a transition from the inflationary epoch approximated by de Sitter space (I) to a radiation-dominated era (R). Suppose that the transition occurs at $\eta=\eta_{1}>0$; then, the scale factor changes as

$$
a(\eta)= \begin{cases}-\frac{1}{H\left(\eta-2 \eta_{1}\right)}, & \text { for }(\mathrm{I})-\infty<\eta<\eta_{1}, \\ \frac{\eta}{H \eta_{1}^{2}}, & \text { for }(\mathrm{R}) \eta_{1}<\eta .\end{cases}
$$

We can expand the metric field $h_{i j}\left(\eta, x^{i}\right)$ in terms of the Fourier modes

$$
a(\eta) h_{i j}\left(\eta, x^{i}\right)=\frac{\sqrt{2}}{M_{\mathrm{pl}}} \frac{1}{\sqrt{V}} \sum_{\boldsymbol{k}} \sum_{A} h_{\boldsymbol{k}}^{A}(\eta) e^{i \boldsymbol{k} \cdot \boldsymbol{x}} p_{i j}^{A}(\boldsymbol{k}),
$$

where $M_{\mathrm{pl}}^{2}=1 /(8 \pi G)$ and a prime denotes the derivative with respect to the conformal time and we introduced the polarization tensor $p_{i j}^{A}(\boldsymbol{k})$ normalized as $p_{i j}^{* A} p_{i j}^{B}=2 \delta^{A B}$. The index $A$ denotes the polarization modes such as $A= \pm$ for circular polarization modes and $A=+, \times$ for linear polarization modes. We note that, since we want to discuss graviton number distribution later, we consider finite volume $V=L_{x} L_{y} L_{z}$ and discretize the $\boldsymbol{k}$ mode $\boldsymbol{k}=$ $\left(2 \pi n_{x} / L_{x}, 2 \pi n_{y} / L_{y}, 2 \pi n_{z} / L_{z}\right)$ with integers $\boldsymbol{n}$.

In quantum field theory, the metric field on the righthand side, $h_{k}^{A}(\eta)$, in Eq. (3) is promoted to the operator. The operator $h_{k}^{A}$ satisfies

$$
h_{k}^{\prime \prime A}+\left(k^{2}-\frac{a^{\prime \prime}}{a}\right) h_{k}^{A}=0
$$

In the inflationary era, the operator $h_{k}^{A}(\eta)$ is then expanded in terms of creation and annihilation operators,

$$
h_{\boldsymbol{k}}^{A}(\eta)=b_{\boldsymbol{k}}^{A} v_{k}^{\mathrm{I}}(\eta)+b_{-\boldsymbol{k}}^{A \dagger} v_{k}^{\mathrm{I} *}(\eta),
$$

where $\left[b_{\boldsymbol{k}}^{A}, b_{\boldsymbol{p}}^{\left.B^{\dagger}\right]}=\delta^{A B} \delta_{\boldsymbol{k}, \boldsymbol{p}}, k\right.$ is the magnitude of the wave number $\boldsymbol{k}$, and $*$ denotes complex conjugation. Equation (4) gives the positive frequency modes $v_{k}^{\mathrm{I}}$ as

$$
v_{k}^{\mathrm{I}}(\eta) \equiv \frac{1}{\sqrt{2 k}}\left(1-\frac{i}{k\left(\eta-2 \eta_{1}\right)}\right) e^{-i k\left(\eta-2 \eta_{1}\right)} .
$$

Similarly, in the radiation-dominated era, we can also expand the operator $h_{k}^{A}(\eta)$ as

$$
h_{k}^{A}(\eta)=c_{k}^{A} v_{k}^{\mathrm{R}}(\eta)+c_{-k}^{A \dagger} v_{k}^{\mathrm{R} *}(\eta),
$$

where $\left[c_{k}^{A}, c_{p}^{B^{\dagger}}\right]=\delta^{A B} \delta_{k, p}$. From Eq. (4), we obtain the positive frequency mode function

$$
v_{k}^{\mathrm{R}}(\eta) \equiv \frac{1}{\sqrt{2 k}} e^{-i k \eta}
$$

Note that $a^{\prime \prime}=0$ for the radiation-dominated era.

The vacuum state for each period is defined as $b_{\boldsymbol{k}}^{A}|0\rangle_{\mathrm{I}}=0, c_{\boldsymbol{k}}^{A}|0\rangle_{\mathrm{R}}=0$. Note that $|0\rangle_{\mathrm{I}}$ is called the BD vacuum. Because the equations of motion for different polarization modes are decoupled in the absence of sources, we focus on either mode below and omit the label of polarization modes $A$ for simplicity unless there may be any confusion. The relation between these different vacua is expressed by a Bogoliubov transformation $b_{k}=$ $\alpha_{k}^{*} c_{k}-\beta_{k} c_{-k}^{\dagger}$, where the Bogoliubov coefficients are calculated as

$$
\alpha_{k}=\left(1-\frac{1}{2 k^{2} \eta_{1}^{2}}-\frac{i}{k \eta_{1}}\right) e^{-2 i k \eta_{1}}, \quad \beta_{k}=\frac{1}{2 k^{2} \eta_{1}^{2}},
$$

so that $\left|\alpha_{k}\right|^{2}-\left|\beta_{k}\right|^{2}=1$ holds. Applying the $b_{k}$ in the Bogoliubov transformation on $|0\rangle_{\mathrm{I}}$ and using $\left[c_{k}, c_{p}^{\dagger}\right]=\delta_{k, p}$, we can express the BD vacuum $|0\rangle_{\mathrm{I}}$ in terms of $c_{k}^{\dagger}, c_{-k}^{\dagger}$ and the vacua associated to each mode, $\left|0_{k}\right\rangle_{\mathrm{R}}$ and $\left|0_{-k}\right\rangle_{\mathrm{R}}$,

$$
|0\rangle_{\mathrm{I}}=\prod_{k} \sum_{n=0}^{\infty} e^{i n \varphi} \frac{\tanh ^{n} r_{k}}{\cosh r_{k}}\left|n_{k}\right\rangle_{\mathrm{R}} \otimes\left|n_{-k}\right\rangle_{\mathrm{R}}
$$

where we defined $\left|n_{k}\right\rangle_{\mathrm{R}}=1 / \sqrt{n !}\left(c_{k}^{\dagger}\right)^{n}\left|0_{k}\right\rangle_{\mathrm{R}}$ and $|0\rangle_{\mathrm{R}}=$ $\left|0_{k}\right\rangle_{R} \otimes\left|0_{-k}\right\rangle_{R}$. The $\varphi$ is an arbitrary phase factor. Here, instead of using the parameter $k \eta_{1}$, we introduced a new parameter $r_{k}$ known as the squeezing parameter, which satisfies tanh $r_{k}=\left|\beta_{k} / \alpha_{k}^{*}\right|$.

The unitary operator to realize Eq. (10) is defined by the squeezing operator

$$
\hat{S}(\zeta)=\exp \left[\zeta^{*} c_{k} c_{-k}-\zeta c_{k}^{\dagger} c_{-k}^{\dagger}\right]
$$

where $\zeta=r_{k} e^{i \varphi}$. Applying the operator $\hat{S}$ to $|0\rangle_{\mathrm{R}}$, we find the resultant state is equivalent to the $\mathrm{BD}$ vacuum [5]. From Eq. (10), we see that the BD vacuum is expressed by a twomode squeezed state of the modes $\boldsymbol{k}$ and $-\boldsymbol{k}$ (or an entangled state) from the point of view of the vacuum in the radiation-dominated era. In this way, particle creation occurs in the course of evolution of the Universe.

Next, we introduce matter fields perturbatively during inflation and see coherent states generated [11].

The coherent state is defined as $b_{k}\left|\xi_{k}\right\rangle_{\mathrm{I}}=\xi_{k}\left|\xi_{k}\right\rangle_{\mathrm{I}}$, where we assumed the eigenvalue $\xi_{k}$ depends only on the magnitude of the wave number. Then, we find a relation, 


$$
\left|\xi_{k}\right\rangle_{\mathrm{I}}=\hat{D}^{\mathrm{I}}(\xi)|0\rangle_{\mathrm{I}}
$$

where we defined the displacement operator

$$
\hat{D}^{\mathrm{I}}(\xi)=\exp \left[\xi_{k} b_{\boldsymbol{k}}^{\dagger}-\xi_{k}^{*} b_{\mathbf{k}}\right] .
$$

Now, let us consider the general action for matter fields. From the definition of energy-momentum tensor $T^{\mu \nu}$, we obtain

$$
S_{\mathrm{m}}=-\frac{1}{2} \int d^{4} x \sqrt{-g} T^{\mu \nu} \delta g_{\mu \nu}+\cdots,
$$

where we considered the linear interaction between metric and the matter field. Hence, the interaction Hamiltonian becomes

$i \int d \eta H_{\mathrm{int}}=\frac{i}{2} \int d \eta \int d^{3} x a^{2}(\eta) h_{i j}(\eta, \boldsymbol{x}) T_{i j}(\eta, \boldsymbol{x})$.

Here, we used Eqs. (3) and (5). This interaction generates a coherent state such as

$$
\begin{aligned}
\left|\xi_{k}^{A}\right\rangle_{\mathrm{I}} & =\exp \left[-i \int d \eta H_{\mathrm{int}}\right]|0\rangle_{\mathrm{I}} \\
& =\prod_{\boldsymbol{k}} \prod_{A} \exp \left[\xi_{k}^{A} b_{\boldsymbol{k}}^{A \dagger}-\xi_{k}^{A *} b_{\boldsymbol{k}}^{A}\right]|0\rangle_{\mathrm{I}},
\end{aligned}
$$

where the coefficients $\xi_{k}^{A}$ are given by

$$
\xi_{k}^{A}=-\frac{i}{\sqrt{2} M_{\mathrm{pl}}} \int d \eta a(\eta) p_{i j}^{A}(\boldsymbol{k}) v_{k}^{\mathrm{I} *}(\eta) T_{i j}(\eta,-\boldsymbol{k}) .
$$

We see that the above state is the same form as Eq. (12). Thus, the BD vacuum in the presence of matter fields becomes a coherent state [12].

\section{GRAVITON STATISTICS}

Now, we characterize nonclassicality by counting graviton numbers $(n)$ in a given state. It is known that the particle number distribution for coherent fields is Poissonian. Because the mean of Poisson distribution is identical to its variance, the Fano factor defined as $F=$ $(\Delta n)^{2} /\langle n\rangle$ equals 1 . Any classical theory leads to the distribution wider than Poissonian (super-Poissonian), and the Fano factor is above 1. Thus, any distribution narrower than Poissonian (sub-Poissonian) or the Fano factor below 1 is a signature of nonclassicality. The point is that these regimes of statistics can be measured with HBT interferometry. Let us calculate graviton statistics of quantum states that the inflationary Universe may have experienced.

We found that the BD vacuum is expressed by a twomode squeezed state from the point of view of the vacuum in a radiation-dominated era. Then, an observer in the vacuum state of radiation-dominated era will observe gravitons defined by the operators $c_{k}$. The expected number of such gravitons will be given by

$$
{ }_{\mathrm{I}}\left\langle 0\left|n_{\boldsymbol{k}}\right| 0\right\rangle_{\mathrm{I}}={ }_{\mathrm{R}}\left\langle 0\left|\hat{S}^{\dagger}(\zeta) c_{\boldsymbol{k}}^{\dagger} c_{\boldsymbol{k}} \hat{S}(\zeta)\right| 0\right\rangle_{\mathrm{R}}={ }_{\mathrm{I}}\left\langle 0\left|n_{-\boldsymbol{k}}\right| 0\right\rangle_{\mathrm{I}},
$$

where we used the relation $|0\rangle_{\mathrm{I}}=\hat{S}(\zeta)|0\rangle_{\mathrm{R}}$ as explained in Eq. (11). Since we cannot distinguish between the modes with $\boldsymbol{k}$ and $-\boldsymbol{k}$, the standard variance is computed as

$$
(\Delta n)^{2}={ }_{\mathrm{I}}\left\langle 0\left|\left(n_{\mathbf{k}}+n_{-\mathbf{k}}\right)^{2}\right| 0\right\rangle_{\mathrm{I}}-_{\mathrm{I}}\left\langle 0\left|n_{k}+n_{-k}\right| 0\right\rangle_{\mathrm{I}}^{2} .
$$

Then, the Fano factor is found to be $F=(\Delta n)^{2} /$ ${ }_{\mathrm{I}}\left\langle 0\left|n_{k}+n_{-k}\right| 0\right\rangle_{\mathrm{I}}=2+2 \sinh ^{2} r_{k}>1$. This shows that the graviton distribution in the BD vacuum is superPoissonian, and we do not see nonclassicality even if measurements of gravitons are made.

Next, let us see the BD vacuum in the presence of classical sources from the point of view of the radiationdominated era. In the presence of matter fields, an observer in a radiation-dominated era will observe gravitons defined by the operators $c_{k}$ in a squeezed coherent state. The expectation number of gravitons can be calculated as

$$
{ }_{\mathrm{I}}\left\langle\xi_{k}\left|n_{k}\right| \xi_{k}\right\rangle_{\mathrm{I}}={ }_{\mathrm{R}}\left\langle\xi_{k}\left|\hat{S}^{\dagger}(\zeta) n_{k} \hat{S}(\zeta)\right| \xi_{k}\right\rangle_{\mathrm{R}}
$$

where we use the relations $\hat{S}^{\dagger}(\zeta) c_{k} \hat{S}(\zeta)=c_{k} \cosh r_{k}-$ $c_{-k}^{\dagger} e^{i \varphi} \sinh r_{k}, \quad c_{k}\left|\xi_{k}\right\rangle_{\mathrm{R}}=\xi_{k}\left|\xi_{k}\right\rangle_{\mathrm{R}}$, and $\xi_{k}=\left|\xi_{k}\right| e^{i \theta}$. The standard variance is also calculated as

$$
(\Delta n)^{2}={ }_{\mathrm{I}}\left\langle\xi_{k}\left|\left(n_{\boldsymbol{k}}+n_{-\boldsymbol{k}}\right)^{2}\right| \xi_{k}\right\rangle_{\mathrm{I}}-{ }_{\mathrm{I}}\left\langle\xi_{k}\left|n_{\boldsymbol{k}}+n_{-\boldsymbol{k}}\right| \xi_{k}\right\rangle_{\mathrm{I}}^{2},
$$

where we assumed that $n_{k}$ and $n_{-k}$ are indistinguishable and calculate the standard variance for the sum of them. In cosmology, we can take $\varphi=0$ in the Bogoliubov coefficients for $k \eta_{1}<1$. Also, we will find $\xi_{k}$ becomes real for two models presented later. Hence, let us consider the case $\theta-\varphi / 2=0$. Then, the Fano factor is simplified as

$$
F=\frac{\left|\xi_{k}\right|^{2} e^{-4 r_{k}}+2 \sinh ^{2} r_{k}+2 \sinh ^{4} r_{k}}{\left|\xi_{k}\right|^{2} e^{-2 r_{k}}+\sinh ^{2} r_{k}} .
$$

Hence, if the Fano factor satisfies the condition $F<1$,

$$
\left|\xi_{k}\right|^{2}\left(e^{-2 r_{k}}-e^{-4 r_{k}}\right)>\sinh ^{2} r_{k}+2 \sinh ^{4} r_{k},
$$

then graviton statistics become sub-Poissonian, and we have a chance to detect nonclassical PGWs with the HBT interferometry in this case.

\section{POSSIBLE DETECTION OF NONCLASSICAL PGWS}

To detect the nonclassical PGWs in the future, we propose making use of the HBT interferometry. 
Originally, HBT interferometry was introduced in the context of radio astronomy [6,7]. They showed that the measurements of intensity-intensity correlations provide accurate measurements of the diameter of stars. In quantum optics, the intensity-intensity correlations are utilized to investigate the nonclassical nature of photons. This concept was first applied to cosmology in Refs. [13-15] and more recently in Ref. [16]. In this work, we aim to make use of the HBT interferometry in order to observe nonclassical PGWs. The HBT interferometer measures intensityintensity correlations, that is, the second-order coherence function defined as

$$
g^{(2)}(\tau)=\frac{\left\langle a^{\dagger}(t) a^{\dagger}(t+\tau) a(t+\tau) a(t)\right\rangle}{\left\langle a^{\dagger}(t) a(t)\right\rangle\left\langle a^{\dagger}(t+\tau) a(t+\tau)\right\rangle},
$$

where the time delay between the signals at the two detectors is expressed by $\tau$. We can rewrite this function in terms of the Fano factor as $g^{(2)}(0)=1+(F-1) /\langle n\rangle$. Thus, $g^{(2)}(0)<1$ if $F<1$. Hence, if the inflationary Universe had experienced a situation in which $F<1$, we would have the chance to detect a nonclassical signature in PGWs with the HBT interferometer. In principle, $g^{(2)}(0)$ can be measured by laser interferometers.

In Refs. [17,18], the authors discuss the measurement performed by the present gravitational wave detectors for the stochastic background. In Ref. [17], the methodology for the two-point correlation is extended to the four-point and higher-order correlations by assuming Gaussian statistics. Note that the intensity-intensity correlation function is a special limit of the four-point correlation function. Hence, if we abandon this assumption and use the temporal four-point correlations, we would be able to measure subPoisson statistics in terms of ground-based and space interferometers.

Now, let us rewrite the condition in Eq. (23) in terms of frequency range of nonclassical PGWs. The squeezing parameter $r_{k}$ can be expressed in terms of frequency at present as follows. We translate the comoving wave number $k$ to the physical frequency $f$ at present as $k / a\left(t_{0}\right)=2 \pi f$, where $t_{0}$ is the present time. By using the conformal time at the moment inflation ends $\eta_{1}$, the parameter $k\left|\eta_{1}\right|$ which corresponds to the squeezing parameter is expressed as $k\left|\eta_{1}\right|=2 \pi f / H\left(t_{0} / t_{\mathrm{eq}}\right)^{2 / 3}\left(t_{\mathrm{eq}} / t_{1}\right)^{1 / 2}$, where the time of matter-radiation equality is given by $t_{\text {eq }}$. Then, we can define the cutoff frequency for the PGWs generated at the end of inflation $f_{1}$ as $k\left|\eta_{1}\right| \equiv f / f_{1}$, where $f_{1}$ is calculated as

$$
f_{1}=10^{9} \sqrt{\frac{H}{10^{-4} M_{\mathrm{pl}}}}(\mathrm{Hz})
$$

and $H$ is the Hubble parameter. Thus, there are no PGWs generated during inflation with frequency higher than the cutoff frequency $f_{1}$. Then, the squeezing parameter is found to be expressed as $\sinh r_{k}=1 / 2 k^{2} \eta_{1}^{2}=\left(f_{1} / f\right)^{2} / 2$. If we consider the PGWs on superhorizon scales $r_{k} \gg 1$, Eq. (23) gives $\left|\xi_{k}\right|^{2}>e^{6 r_{k}} / 8$, and the squeezing parameter becomes $e^{r_{k}}=f_{1}^{2} / f^{2}$. Combining these equations, we find the condition to detect nonclassical PGWs (23) can be approximately written as

$$
f>\left(\frac{1}{8}\right)^{\frac{1}{12}} 10^{9}\left|\xi_{k}\right|^{-\frac{1}{6}} \sqrt{\frac{H}{10^{-4} M_{\mathrm{pl}}}}(\mathrm{Hz}) .
$$

Since $1 \mathrm{GHz}$ is a cutoff scale for PGWs generated during inflation, we have the chance to detect the nonclassical PGWs if the amplitude of $\left|\xi_{k}\right|$ is larger than 1.

To predict the frequency range of nonclassical PGWs explicitly, we estimate $\left|\xi_{k}\right|$ of Eq. (26) by considering two models with a gauge field as the classical matter fields. In both modes, gauge fields grow during inflation and disappear after the inflation.

\section{A. Anisotropic inflation}

The model to realize anisotropic inflation $[19,20]$ is given by

$\mathcal{L}=\frac{M_{\mathrm{pl}}^{2}}{2} R-\frac{1}{2}\left(\partial_{\mu} \phi\right)\left(\partial^{\mu} \phi\right)-U(\phi)-\frac{1}{4} J^{2}(\phi) F_{\mu \nu} F^{\mu \nu}$,

where $U(\phi)$ and $J(\phi)$ are arbitrary potential and coupling functions that satisfy a relation $J(\phi)=$ $\exp \left[q / M_{\mathrm{pl}}^{2} \int d \phi U(\phi) / \partial_{\phi} U(\phi)\right]$. Here, $q$ is a dimensionless parameter. The equation of motion for the gauge field $A_{i}(\eta, \boldsymbol{k})$ satisfies

$$
A_{i}^{\prime \prime}(\eta, \boldsymbol{k})+2 \frac{J^{\prime}}{J} A_{i}^{\prime}(\eta, \boldsymbol{k})+k^{2} A_{i}(\eta, \boldsymbol{k})=0 .
$$

The solutions of the electric and magnetic fields are given in terms of the gauge fields

$E_{i}(\eta, \boldsymbol{k})=-J \partial_{\eta} A_{i}(\eta, \boldsymbol{k}) \simeq \sqrt{p}\left(\frac{1}{p \eta}\right)^{\nu} \gg B_{i}(\eta, \boldsymbol{k})$,

and this contributes to $T_{i j}$ in Eq. (17). Then, we find

$$
\left.\xi_{k} \simeq \frac{\sqrt{V p^{3}}}{M_{\mathrm{pl}}} E_{i}^{2}(\eta, \boldsymbol{p})\right|_{p \sim k},
$$

where we used Eq. (6) for $k \eta \ll 1$ and estimated the generation of gravitons for the duration of inflation and then replaced the time integral by $1 / H$, which should be the minimum estimation. We also regarded the volume $V$ is large enough and replaced the summation with respect to $\boldsymbol{p}$ by integration. We then simply approximated it by $p^{3}$ on 
dimensional grounds. Since $E_{i}$ and $B_{i}$ are rapidly oscillating, we can approximate $\boldsymbol{k} \simeq \boldsymbol{p}$.

The duration of the growth of gauge fields is given by the number of $e$-foldings $N_{\text {gauge }}=-\log (-p \eta)$. Thus, the electric field of the solution in Eq. (29) can be written as

$$
E_{i}(\eta, \boldsymbol{p}) \simeq \sqrt{p} e^{\nu N_{\text {gauge }}}
$$

where $\nu \sim q U /\left(M_{\mathrm{pl}} \partial_{\phi} U\right)$. It would be legitimate to consider $V$ as an observable region, that is, $V \sim 1 / H_{0}^{3}$, where $H_{0}$ is the Hubble constant at present. Then, if we write $p=2 \pi f \sim f$, we obtain

$$
\left|\xi_{k}\right|^{2} \simeq 10^{19}\left(\frac{f}{1 \mathrm{GHz}}\right)^{5} e^{4 \nu N_{\text {gauge }}}
$$

Plugging this into Eq. (26), we find

$$
f>10^{8.1} e^{-\frac{4}{17} \nu N_{\text {gauge }}}\left(\frac{H}{10^{-4} M_{\mathrm{pl}}}\right)^{\frac{6}{17}}(\mathrm{~Hz}) .
$$

Hence, we can detect nonclassical PGWs for $f>100 \mathrm{kHz}$ with $\nu N_{\text {gauge }} \sim 30$ and $H=10^{-4} M_{\mathrm{pl}}$. The boundary of the frequency range of nonclassical PGWs for anisotropic inflation model is depicted by a dotted-dashed green line in Fig. 1. As was shown in Refs. [21,22], the density parameter of PGWs $\Omega_{\mathrm{GW}}$ can be sufficiently large depending on the model parameter.

\section{B. Axion inflation}

In the axion model [23-27], an axion field $\phi$ couples to gauge fields and experiences a tachyonic instability. This instability leads to the exponential growth of gauge fields.

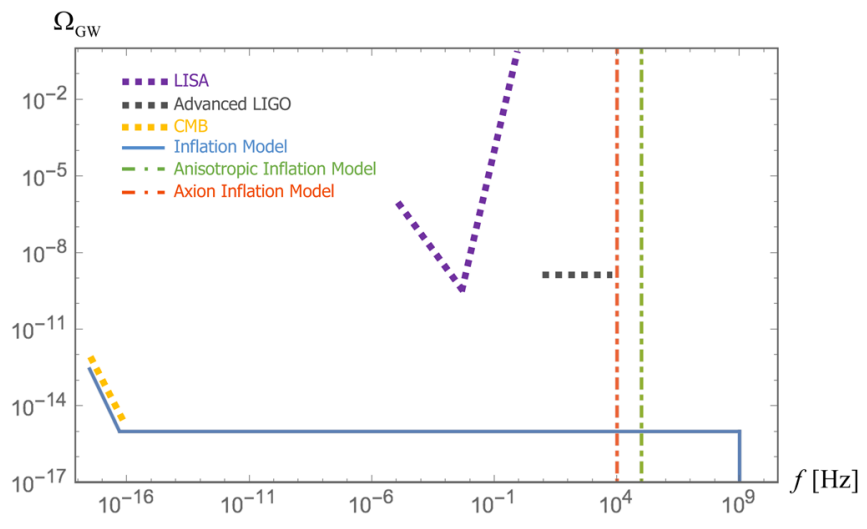

FIG. 1. Normalized GW energy density vs frequency. The frequency range in which we can detect nonclassicality is higher than $10^{5} \mathrm{~Hz}$ (dotted-dashed green) for the anisotropic inflation model and higher than $10^{4} \mathrm{~Hz}$ (dotted-dashed red) for the axion inflation model.
The model we consider is

$$
\begin{aligned}
\mathcal{L}= & \frac{M_{\mathrm{pl}}^{2}}{2} R-\frac{1}{2}\left(\partial_{\mu} \phi\right)\left(\partial^{\mu} \phi\right)-U(\phi)-\frac{1}{4} F_{\mu \nu} F^{\mu \nu} \\
& -\frac{\lambda}{8 f_{a}} \phi \epsilon^{\mu \nu \alpha \beta} F_{\mu \nu} F_{\alpha \beta},
\end{aligned}
$$

where $f_{a}$ is an axion decay constant, $\lambda$ is a dimensionless parameter, and $\epsilon^{\mu \nu \alpha \beta}$ is the Levi-Cività tensor. The potential $U(\phi)$ is the conventional cosine potential.

The gauge field obeys the following equation of motion:

$$
\left[\frac{\partial^{2}}{\partial \eta^{2}}+k^{2} \pm \frac{2 k \chi}{\eta}\right] A_{i}^{ \pm}(\eta, \boldsymbol{k})=0, \quad \chi \equiv \frac{\lambda \phi^{\prime}}{2 a f_{a} H} .
$$

In this model, we have a classical source,

$$
E_{i}(\eta, \boldsymbol{k}) \simeq B_{i}(\eta, \boldsymbol{k}) \simeq \frac{a H}{\sqrt{k}}\left(\frac{k}{\chi a H}\right)^{\frac{1}{4}} e^{\pi \chi} .
$$

Thus, we obtain

$$
\left|\xi_{k}\right|^{2}=\frac{k}{H_{0}} \frac{a^{4} H^{4}}{M_{\mathrm{pl}}^{2} H_{0}^{2}}\left(-k \eta_{1}\right) \frac{e^{4 \pi \chi}}{\chi}
$$

This can be translated into the frequency range in which we can detect the nonclassicality

$$
f>10^{7.9} e^{-\frac{2}{7} \pi} \chi \chi^{\frac{1}{14}}\left(\frac{H}{10^{-4} M_{\mathrm{pl}}}\right)^{\frac{9}{28}}(\mathrm{~Hz})
$$

For example, if we take $\chi \sim 10$ and $H=10^{-4} M_{\mathrm{pl}}$, this reduces to $f>10 \mathrm{kHz}$. Namely, we can marginally detect nonclassicality in the PGWs with the LIGO detector. The boundary of the frequency range of nonclassical PGWs for the axion inflation model is depicted by the dotted-dashed red line in Fig. 1.

Regarding sensitivity, we note here that the current gravitational wave detectors measure the amplitudeamplitude correlations (two-point correlations) and we need to measure the intensity-intensity correlations (fourpoint correlations) in order to make use of the HBT interferometry. In principle, there would not be big difference in methodology from the current detectors, although we would need more sensitivity to detect PGWs. As for the detection of gravitational waves with high frequency, we would need gravitational wave detectors with smaller arms.

\section{CONCLUSION}

We considered the possible detection of nonclassicality of PGWs by applying HBT interferometry to cosmology. We characterized the nonclassicality of PGWs in terms of sub-Poissonian statistics and the Fano factor below 1, as is often done in quantum optics. We showed that the initial 
presence of classical sources induces coherent states during inflation, and the Bunch-Davies vacuum looks like squeezed coherent states from the point of view of the radiationdominated era. We calculated graviton statistics of the squeezed coherent states and showed that it becomes subPoissonian. We proposed the HBT interferometry to detect the nonclassical PGWs and gave a criterion for the nonclassicality in terms of the frequency of the PGWs in Eq. (26). To predict the frequency range of nonclassical PGWs, we presented two concrete models, namely, the anisotropic inflation model due to gauge fields and the axion inflation model with Chern-Simons coupling. We found that if the PGWs with a frequency range higher than $100 \mathrm{kHz}$ for anisotropic inflation or $10 \mathrm{kHz}$ for axion inflation model were detected, the PGWs could tell us information about the quantum mechanical origin of the Universe.

There are many directions we can pursue. We can extend our analysis to PGWs with polarizations in terms of Stokes parameters. This would be useful to narrow down the inflation models that produce PGWs with polarizations
[28-32]. Recently, it was shown that there exists entanglement with causally disconnected regions [33-48]. If we consider the effect of entanglement on the initial quantum state, we may be able to prove the existence of other universes by the nonclassical PGWs. Although we assumed that gravitons travel through the Universe virtually unimpeded, we need to clarify the effect of decohenrece on graviton statistics in the future $[49,50]$. Needless to say, it is essential to develop a more concrete HBT method to detect nonclassical PGWs.

\section{ACKNOWLEDGMENTS}

S. K. was supported by JSPS KAKENHI Grant No. JP18H05862. J.S. was in part supported by JSPS KAKENHI Grants No. JP17H02894, No. JP17K18778, No. JP15H05895, No. JP17H06359, and No. JP18H04589. J. S. is also supported by JSPS Bilateral Joint Research Projects (JSPS-NRF Collaboration) "String Axion Cosmology."
[1] B. P. Abbott et al. (LIGO Scientific and Virgo Collaborations), Phys. Rev. Lett. 116, 061102 (2016).

[2] S. Kawamura et al., Classical Quantum Gravity 28, 094011 (2011).

[3] P. Amaro-Seoane et al., GW Notes 6, 4 (2013).

[4] A. Maleknejad, M. M. Sheikh-Jabbari, and J. Soda, Phys. Rep. 528, 161 (2013).

[5] G.S. Agarwal, Quantum Optics (Cambridge University Press, Cambridge, England, 2012).

[6] R. H. Brown and R. Q. Twiss, Nature (London) 178, 1046 (1956).

[7] R. H. Brown and R. Q. Twiss, Nature (London) 177, 27 (1956).

[8] D. Polarski and A. A. Starobinsky, Classical Quantum Gravity 13, 377 (1996).

[9] P. A. R. Ade et al. (Planck Collaboration), Astron. Astrophys. 594, A20 (2016).

[10] S. Koh, S. P. Kim, and D. J. Song, J. High Energy Phys. 12 (2004) 060.

[11] This is in contrast to the situation discussed in C. Kiefer, D. Polarski, and A. A. Starobinsky, Phys. Rev. D 62, 043518 (2000).

[12] R. J. Glauber, Phys. Rev. 130, 2529 (1963).

[13] M. Giovannini, Phys. Rev. D 83, 023515 (2011).

[14] M. Giovannini, Classical Quantum Gravity 34, 035019 (2017).

[15] M. Giovannini, Mod. Phys. Lett. A 32, 1750191 (2017).

[16] J. W. Chen, S. H. Dai, D. Maity, S. Sun, and Y. L. Zhang, Phys. Rev. D 99, 023507 (2019).

[17] B. Allen and J. D. Romano, Phys. Rev. D 59, 102001 (1999).
[18] B. P. Abbott et al. (LIGO Scientific and Virgo Collaborations), Phys. Rev. Lett. 120, 201102 (2018).

[19] M. a. Watanabe, S. Kanno, and J. Soda, Phys. Rev. Lett. 102, 191302 (2009).

[20] J. Soda, Classical Quantum Gravity 29, 083001 (2012).

[21] K. Choi, K. Y. Choi, H. Kim, and C. S. Shin, J. Cosmol. Astropart. Phys. 10 (2015) 046.

[22] A. Ito and J. Soda, J. Cosmol. Astropart. Phys. 04 (2016) 035.

[23] N. Barnaby and M. Peloso, Phys. Rev. Lett. 106, 181301 (2011).

[24] N. Barnaby, R. Namba, and M. Peloso, J. Cosmol. Astropart. Phys. 04 (2011) 009.

[25] J. L. Cook and L. Sorbo, Phys. Rev. D 85, 023534 (2012); 86, 069901(E) (2012).

[26] M. M. Anber and L. Sorbo, Phys. Rev. D 85, 123537 (2012).

[27] N. Barnaby, E. Pajer, and M. Peloso, Phys. Rev. D 85, 023525 (2012).

[28] M. Satoh, S. Kanno, and J. Soda, Phys. Rev. D 77, 023526 (2008).

[29] T. Takahashi and J. Soda, Phys. Rev. Lett. 102, 231301 (2009).

[30] I. Obata, and J. Soda, Phys. Rev. D 93, 123502 (2016); 95, 109903(A) (2017).

[31] M. a. Watanabe, S. Kanno, and J. Soda, Prog. Theor. Phys. 123, 1041 (2010).

[32] T. Fujita, I. Obata, T. Tanaka, and S. Yokoyama, J. Cosmol. Astropart. Phys. 07 (2018) 023.

[33] J. Maldacena and G. L. Pimentel, J. High Energy Phys. 02 (2013) 038. 
[34] S. Kanno, J. Murugan, J. P. Shock, and J. Soda, J. High Energy Phys. 07 (2014) 072.

[35] N. Iizuka, T. Noumi, and N. Ogawa, Nucl. Phys. B910, 23 (2016).

[36] N. Bolis, A. Albrecht, and R. Holman, J. Cosmol. Astropart. Phys. 12 (2016) 011; 08 (2017) E01.

[37] S. Kanno, M. Sasaki, and T. Tanaka, J. High Energy Phys. 03 (2017) 068.

[38] A. Matsumura and Y. Nambu, Phys. Rev. D 98, 025004 (2018).

[39] S. Choudhury and S. Panda, Eur. Phys. J. C 78, 52 (2018).

[40] S. Choudhury and S. Panda, arXiv:1712.08299.

[41] A. Higuchi and K. Yamamoto, Phys. Rev. D 98, 065014 (2018).
[42] S. Kanno, J. P. Shock, and J. Soda, J. Cosmol. Astropart. Phys. 03 (2015) 015.

[43] S. Kanno, J. P. Shock, and J. Soda, Phys. Rev. D 94, 125014 (2016).

[44] D. Campo and R. Parentani, Phys. Rev. D 74, 025001 (2006); Braz. J. Phys. 35, 1074 (2005).

[45] J. Maldacena, Fortschr. Phys. 64, 10 (2016).

[46] J. Martin and V. Vennin, Phys. Rev. A 93, 062117 (2016).

[47] S. Choudhury, S. Panda, and R. Singh, Eur. Phys. J. C 77, 60 (2017).

[48] S. Kanno and J. Soda, Phys. Rev. D 96, 083501 (2017).

[49] C. Kiefer and D. Polarski, Adv. Sci. Lett. 2, 164 (2009).

[50] A. Albrecht, S. Kanno, and M. Sasaki, Phys. Rev. D 97, 083520 (2018). 